\title{
Sending Shivers Down the Spine. VR Productions as Seamed Media
}

\author{
Ágnes Karolina Bakk \\ Moholy-Nagy University of Art and Design Budapest (Hungary) \\ Sapientia Hungarian University of Transylvania (Cluj-Napoca, Romania) \\ E-mail: bakkagnes@gmail.com
}

\begin{abstract}
According to Rebecca Rouse's concept of "media of attraction” (2016), the mediums of virtual reality have four characteristics: they are participatory, interdisciplinary, unassimilated and seamed. The author's hypothesis is that even though 360-degree films and virtual reality experiences as seamed mediums are remediating the medium of film, they have the characteristics of the medium of live performance. She points out that the characteristics of performance art based on Fischer-Lichte's taxonomy (2008), such as liveness and co-presence, are influencing the development of 360-degree films and virtual reality experiences. As an argument, she analyses three virtual reality productions created by performing artists, which operate with the specificity of intermediality and the longing for immersion, the main characteristic of virtual reality. These productions lean on the immediacy characteristics of the medium of film and performance by using cut-scenes, linear narratives, live streaming, but also by including the "human interface," i.e., the actor, who ensures a higher level of absorption. ${ }^{1}$
\end{abstract}

Keywords: virtual reality, immersion, performing arts, liveness, intermediality.

Before discussing the Virtual Reality productions created by performing arts companies, it is important to define the difference between 360-degree movie and VR experience. In everyday language, VR movie can designate 360-degree videos as well as computer generated VR experience with live rendering. 360-degree videos are situating the spectator in the place of the camera, which is a passive position similar to that of film viewers. The immersive characteristic of this genre consists in that the environment can be explored in 360 degrees by head movements, but staying in a fixed position. 360 degree-videos (unless they are interactive), therefore,

1 This work was supported by a grant of the Ministry of National Education, CNCS - UEFISCDI Romania, project number PN-III-P4-ID-PCE-2016-0418. 
have a fixed storyline and timeline, and thus, they also have a predefined viewing time and the narrative is controlled by the creators. In contrast, computer generated VR environments offer viewers an experience that leads to a more participative attitude. These are computer-generated environments that can be explored by the viewer, that is, game-based environments where the players are the protagonists and they are making the choices - as long as that is enabled by the design. The two genres are named in the similar way because, for a better viewing experience, both of them should be watched with the use of goggles or head-mounted displays.

As the two production types (that can also be considered as two different genres) are both labelled "virtual reality," it is important to point out that only VR experiences enable in themselves interactivity for the participants. Therefore, we can state that even though both of these main types of productions can be experienced in their full by using the headset, 360-degree videos are more related to the medium of film, while VR experiences, which give more opportunities for the user to get into interactive relations with the immersive space, are more based on video game mechanisms. In video game mechanics, game designers basically have to anticipate player strategies. Game designers are using clues, sounds, and dramatic actions to guide the players. Such game mechanics can be used in theatrical performances that combine VR and the bodily co-presence of the audience with the performers, and in this case, they can create various emotions, or can also highlight the "ecstasies of things," to borrow a phrase from Böhme (1995, 121). According to Fischer-Lichte, this phrase describes "the presence of objects more accurately. Presence brings forth humans as what they already are: embodied minds. Ecstasy, in turn, makes things appear as what they already are but usually remain unnoticed in everyday life because of their instrumentalization" $(2008,100)$. The objects that can be used in productions where real time events are combined with VR experiences do have this "ecstatic" character as they receive more layers of possible interpretations.

\section{Performing Art Using Virtual Reality}

As early as 1938, Antonin Artaud called theatre virtuel realité in his book The Theatre and Its Double. Already by the mid-20th century, theatre professionals started to be interested in the concept of virtual reality. If we consider virtual reality as an illusion which can take over the audience immediately, it is important to have a look at how performance ${ }^{2}$ is introducing the promise of virtual reality into its

2 The notions of theatre performance and performance have been differentiated by Fischer-Lichte 
practice, the promise of transporting the viewer into another space. Today, there are several theatre companies using 360-degree video technology. The most widespread use is the 360-degree live streaming, which began with Broadway productions such as The Lion King (2015). Many performance companies are now using 360-degree video to engage their viewers by promising them a new perspective of being on the stage and the excitement of liveness. Dance and even circus companies are creating their VR movies, partly with advertisement goals. In 2016, the Dutch National Ballet created Night Fall, the first virtual reality ballet in the world. The performance takes place in a rehearsal room where we can also see renowned dancers like Anna Tsygankova. The spectators have the feeling that they are sitting in the middle of the room while the dancer and the violinist are moving around them. Cirque du Soleil also created several 360-degree movies, based on their already famous performances such as $K \grave{A}$ (2016), Dreams of 'O' (2017), etc., ${ }^{3}$ and their production company has also released a separate mobile phone app for these 360-degree movies.

In many of these performances, the viewers have the feeling of being part of what is happening on the stage, but they cannot influence it because of the lack of an interactive aspect. Also, these productions are ephemeral. They are live streamed, and their fame is spread usually via social networks, and because their attractiveness for the audiences depends on the novelty of the technology, many of these productions enjoy just a short period of fame and do not get established as classics.

However, there have also been production companies which integrate the use of VR head-mounted displays into their practice by enhancing it with various aspects of performance, such as Punchdrunk or Bombina Bombast. In their production, Believe Your Eyes, shown only to 500 people at Miami Art Week in 2016, Punchdrunk synchronized in an uncanny way the rendered image screened inside the head-mounted display with the live actions and presence. The audience was ushered by an actress into an isolated spot and asked to wear a helmet which incorporated a head-mounted VR display. The 4D visual presentation featured the same actress moving around the room. Based on the reports about the performance, the actress was present in the room, she was speaking to the participants, letting them feel her breath or touching them actress and audience remaining in this way incredibly "close" throughout the performance (Graver 2016).

(2008). She attributes to performance characteristics such as liveness and co-presence, which have a transformative power.

For the full list, see: www.felixandpaul.com. Last accessed 21. 07. 2019. 
In 2017, CyberRäuber created a performance called Der Geisterseher (The Ghost-Seer) that premiered in Mannheim at Schillertage Festival. It was based on Der Geisterseher, a lesser-known novel by Friedrich Schiller, a first-person narrative of a prince named O, taking place at the time of a Venice Carnival. The story has elements of gothic novels, and also spiritualism, necromancy and unfulfilled love. However, in CyberRäuber's theatrical show, this fragmented narrative was not constructed from a single syuzhet, rather, a series of elements came together in this 360-degree production and brought it closer to the realm of VR. The physical setting for the audience to step into this world was a motion tracked space, where one could put on the head-mounted display and watch the 360-degree animation, made with a program called Unity. The production was pre-scripted, there was no possibility for interaction, but the participant could move freely in a small space, as the motion track camera enabled it. The 360-degree production was directed by a theatre director. The protagonist, the prince, talked directly to the viewer at the beginning, but later the viewer became only the witness of various private discussions. The set design was constructed to resemble the inside of a gothic cathedral, where the viewer had the ability to move through a limited space only, offered by the motion tracking system. The characters in the virtual space, animated via the movements of physical actors, surrounded the viewers, sometimes even going through their bodies. The characters sometimes seemed to vibrate, with the graphics often deconstructing themselves, uncovering the technological constructedness of the representation. The 360-degree video's length is 17 minutes, with a fixed line of sequences.

In The Shared Individual (2016), the production of the Swedish theatre group Bombina Bombast\&Makropol the spectators can experience a unique interaction between viewers and the medium of VR by playing along with the visual isolation of the head-mounted display. In their performances, they ask each member of the audience to put on a headset, then one of the members of the group sits in front of them with the same headset, but on this latter there are several cameras mounted that livestream the audience's current actions. Thus, in their virtual space, the participants see themselves via the camera that is installed on the performer's headset. First, the artists verbally perform a meditative embodiment exercise: they ask the audience to synchronize physically with the performer and to mimic the performer's movements, and then also to synchronize "with her soul." The series of acts end with a joke that reflects on this ritual-like exercise with some irony. Suddenly, in their virtual space an empty row of seats appears instead of their own image - the participants' seats filmed prior to the show and sequenced 
into the performance using the technique of montage. Then the participants start to create interactive situations, which involves a self-identifying feedback-loop. Through this process they are trying to bridge the gap caused in their perception of their spatial positions and identity by the rapid montage. With this performance, the creators question the location of the stage during a VR performance.

\section{Theoretical Assumptions}

In the previous section, I went through several examples of performances that feature the technique of VR head-mounted display, and tried to demonstrate how these productions simply rely on the attraction of a new technology, or also incorporate aspects of performance. In this section, I will point out how these performances using VR head-mounted displays depend on the one hand on the model of cinema and how, on the other hand, they are using human interactivity (or human interface) to make the immersive aspect of VR productions more vivid relying on the "seamed character" of these productions.

As Bolter and Grusin state, the medium of film is an integral part of VR. Even though VR has attempted to develop as a unique media, it could not "do away with film, television, and other media precisely because there would then be no standards against which to judge its remediations. [...] One way to understand virtual reality, therefore, is as a remediation of the subjective style of film, an exercise in identification through occupying a visual point of view" (Bolter and Grusin 2000, 163). Our experiences with VR are not free from a reference to our past experiences with film. However, as we have seen in the case of Bombina Bombast and Punchdrunk, there are also performances that feature VR experiences in a performative context that does not necessarily rely only on the media tactics of cinema. In the aforementioned productions where the audience is instructed to put on a head-mounted display, the effect of immersion is much stronger when the audience puts on the VR devices as part of a performance the plot of which they take part in. The sense of immersion is made possible via the transparency of the medium, the transparency of the screen of the VR device, which makes possible the immediacy of the content. One could argue that participating in a performance as a character who is wearing a VR device would actually decrease the effect of immersion, as the spectator is conscious of wearing a device. However, we should not forget that in these performances, the members of the audience put on the VR devices via performative acts that can be considered as enactments of ceremonies, therefore increasing the effect of 
immersion for the audience. VR productions borrow from immersive theatre the different rituals of how they let their users enter their world.

Therefore, even though virtual reality productions (either 360-degree movies, or VR experiences) offer immediacy - this is why some creators such as Chris Milk (2015) call it the "ultimate medium" -, they need the framing of an interactive situation that enhances the immediate immersion the participants encounter when putting on the head-mounted display. This particular moment in history, when VR is getting impregnated by elements of performance, gives us a reason to interpret VR as still having the status of a "seamed medium."

As Rebecca Rouse (2016) framed the meta-category of "media of attraction" based on Gunning's concept of "cinema of attraction," she suggested to include into these categories interactive works created by using virtual reality or augmented reality techniques. The central qualities that such artworks should have are the following: participatory, interdisciplinary, unassimilated (i.e., not yet institutionalized) and seamed. Rouse writes about this latter: "because of this rich multiplicity of design approaches and structures exhibited by media of attraction, these media are not 'seamless' as technology industry rhetoric might have us believe. Instead, the media of attraction are decidedly seamed. Edges show between parts of a media of attraction experience, and the patchwork of ways in which multiple forms of representation come together are not hidden from spectators" (Rouse 2016, 101). In the cases mentioned above, the VR productions can be considered seamed as they show the importance of the performative mechanism that helps the user to step into the immediate immersive world - as in the case of Punchdrunk and Bombina Bombast. In the production of CyberRäuber this mechanism is not used, the viewer is stepping into this immersion by putting on the head-mounted display, and has no other direct interaction possibility, such that a human interface co-presence could offer. On the other hand, Marie-Laure Ryan states: "VR is not so much a medium in itself, as a technology for synthesis of all media toward a total experience" $(1999,112)$. Thus she draws attention to the fact that VR interface is so transparent that in fact "the 'virtual reality effect' is the denial of the role of signs (bits, pixels, and binary codes) in the production of what the user experiences as unmediated presence" (Ryan 1999, 112). This transparency of the interface, which comes with the price of the disappearance of the semiotic properties of the artwork, is very much connected to the creation of a self that emerges as a result of the subject being immersed in the artwork.

Chris Salter's recommendation is to expand on Foucault's four technologies "that train, produce and regulate modern selves" (2017): (1) technologies of 
production, (2) technologies of sign systems, (3) technologies of power, (4) technologies of the self (Foucault 1982). Salter introduces the fifth "technology" that he names "technologies of sense" and defines as the techniques, devices, procedures or strategies that aim to produce bodies and selves with other kinds of perceptions that "extend routine ways of seeing, hearing, feeling, touching and tasting the world" $(2007,175)$. In the category of the fifth technology, we can also include VR technologies, especially due to their live rendering and immersive characteristics, but also because of the already mentioned "ecstasy of things."

This capability of VR's medium of total immersion is also due to the characteristics of creating a strong atmospheric feeling in such productions. "Atmosphere" is another concept that can help us here in analysing the performances that intersect with VR. The above-mentioned performative acts all involve an interactive situation, and all interactive situations require a context which we can call an "atmosphere." For Fischer-Lichte, atmosphere is the fundamental constitutive level in a performance that creates the sense of immersion. "Since atmosphere is constituted both by the actors' presence as well as the ecstasy of things, it impresses itself particularly intensely onto the perceiving subjects. Atmosphere envelops the subjects who become immersed in them, penetrating the subjects' bodies as light, sounds, or odors." (Fischer-Lichte 2008, 154.) This echoes Böhme's ideas: "atmosphere is the common reality of the perceiver and the perceived. It is the reality of the perceived as the sphere of its presence and the reality of the perceiver, insofar as in sensing the atmosphere s/ he is bodily present in a certain way" $(1993,122)$.

It is also helpful to borrow the philosophical concept of possible worlds, a concept applied to the literary theory by theorists like Eco (1984), Doležel (1998), and others. In a semantic model that contains a plurality of worlds, there is only one that we consider as the actual world, the one that exists independently of our human mind, the others are non-actual worlds that are constructed by different activities of human mind, like dreaming, imagining. According to Ryan, "VR adds to this catalog of 'accessibility relations' a mode of apprehension that involves not only the mind, but also the body. For the first time in history, the possible worlds created by the mind become palpable entities, despite their lack of materiality" (1999, 115).

Although the mechanics of stepping into VR worlds might sometimes seem too abrupt and can cause bodily reactions such as nausea, dizziness and coordination problems, this could be transformed into a performative act, while the subjects of the VR experience can slowly adapt themselves to the experience. Performance 
can contribute here by smoothing and contextualizing this performative act with live, bodily co-presence of an actor. This co-presence effect enhances with liveness the atmosphere of the VR production, no matter what the filmed settings actually look like.

Creating this common reality through the feedback loop between the audience and the performers is an aim of performance artists. According to FischerLichte, performances aim at the involvement of all participants, in order to create a reciprocal relationship of influences. She believes that "the feedback loop thus identifies transformation as a fundamental category of an aesthetics of the performative" $(2008,50)$ and explains that "the feedback loop as a selfreferential, autopoietic system enabling a fundamentally open, unpredictable process emerged as the defining principle of theatrical work. [...] it needs to be investigated how actors and spectators influence each other in performance; what the underlying conditions of this interaction might be; what factors determine the feedback loop's course and outcome; and whether this process is primarily social rather than aesthetic in nature" $(2008,29)$. Katja Kwastek further develops the concept of an autopoietic feedback loop (a concept borrowed from biology by Fischer-Lichte [2008]). For her, this feedback loop is different than the feedback processes in interactive media art, in technical systems that "feedback process" cannot be translated to face-to-face interaction (Kwastek 2013, 99). She argues that "ontologically, interactive media art can therefore be compared with an apparatus. Apparatuses unite presentability and performability in the sense that they are at one and the same time manifest entity, invitation to act, and basis for performance. [...] Interactive media artworks are comparable to apparatuses, although their aesthetic potential should primarily be sought in the realization of the processes they enable. However, this doesn't exclude the possibility that this realization may also turn into a creative activity, within which the interaction system will become a facilitator for the creation of manifestations - which in turn can become the object of a contemplative observation" (Kwastek 2013, 262).

Indeed, VR experiences (as in the case of Bombina Bombast's productions) can become such "facilitators," but only by using the performativity and the co-presence of performance. In interactive art (including VR experiences), artists need not be present as authors, they can also be present in other roles, as observers, mediators or players (Kwastek 2013, 93). In both Bombina Bombast and Punchdrunk's performances, live human interface is co-present, either as an actor or as a mediating actor of the performance. In Bombina Bombast's The Shared Individual the creators are experimenting with how a process of embodiment 
unfolds. As Erika Fischer-Lichte puts it: "previously the actor's movements were designed to translate meaning laid down in the literary text. Now they served as a stimulus to induce excitement in the spectators and/or motivate them to generate new meaning themselves. While the first enlisted performativity in the service of expressivity, it was now seen to possess an energetic, affective potential" (2008, 82). In Bombina Bombast and Punchdrunk's productions the bodily co-presence of the actor has such an affective potential on the viewer.

We can conclude that Fischer-Lichte's concept of an autopoietic feedbackloop can be applied only to VR productions like Punchdrunk's or Bombina Bombast's productions that include elements of performance. The Ghost-Seer does not really present such features as it mainly relies on elements of cinematic narrative experienced in a more passive way as the participants' movement in space is not changing the actual plot. In Punchdrunk's case, the participants can already feel by the bodily co-presence that they are the vulnerable subjects of the performance and they feel the "unpredictability" of the feedback loop (see Fischer-Lichte 2008, 47). In the feedback loop system, the constant disruptions can be pointed out mainly in Bombina Bombast's production: here the audience does not have automatic reactions, they are continuously forced to make new decisions whether to accept what is happening on the stage or not, and also by taking their decision they affect the other audience member's experience.

As Fischer-Lichte $(2018,17)$ demonstrates with performances such as those of Marina Abramovic, the spectators in a performance can be involved in a common situation of here and now, transforming everyone present into cosubjects. Similarly, we can state that Bombina Bombast's performance explored liveness similarly and created a performative artwork through the immersive character of the production. The performer who orchestrated this immersive experience was orchestrating different illusionary "hacks" enabled by the VR set and the sensorial experience created by the apparatus. It is important to note that in their case the space in which the action took place was playfully using the reflection of the real space in the illusionary space. The performance created an uncomfortable and uncanny experience for the audience members, which was full of possibilities of exploration, and did this by combining the virtual space with the live space through rapid changes. This resembled the technique of montage as a characteristic of the medium of film.

While the creators working with CyberRäuber simply adapted a non-theatrical text to a $3 \mathrm{D}$-video production, in the case of the other two productions, the creators having a background in performing arts, employed another kind of 
"media tactics" and adapted to VR the immediacy of theatre. By the combination of the use of "human interface" with the ritualistic situation of taking on the virtual reality headset, participants can be part of an intiation ceremony, a rite of passage. Switching between worlds and between immersive situations, the act of starting or finishing a VR production as well as the movements that are accompanying it, all have a performative effect similar to a rite of passage.

Although, as mentioned above, "atmosphere," "performativity" and "interaction" can be key characteristics in describing experiences, in the context of VR-experiences these mainly depict key moments of the works, and not the whole experience as such. Taking into consideration the common reality of the perceiver and the perceived, who interact within the same atmosphere, Giannachi and Benford (2011) propose that mixed-reality performances that use hybrid spaces, time, interfaces and roles can be understood in terms of trajectories. The key aspect to their theory is that "mixed reality performances can be understood in terms of three fundamental types of trajectory: canonical trajectories express artists' intended narratives or journeys through a work; participant trajectories express the actual journeys that different individuals undertake; and historic trajectories synthesize different views of what took place as the experience is subsequently replayed" $(2011,206)$. Trajectory, in this sense, can also be a fruitful term in the analysis of VR productions, as it includes both the planned and the "lived" experiences. We can state, for example, that while the trajectory of the Ghost-Seer is very unilateral, as it lacks almost any interactivity (there is only the participants' movement in the space), Punchdrunk and Bombina Bombast are different in this respect. Punchdrunk is a single-person experience, so the trajectory of the participant can be more under the control of the live actor (who can also be a so-called orchestrator, the person who helps put on the helmet). With Bombina Bombast, the experience depends very much on the audience, who this way become the participants.

\section{Using Liveness in VR Productions}

The ideal promise of immersion is to transport the observer into another space or world, creating a special sense of telepresence (see Grau 2003; Griffiths 2008). This is what many art forms strived for throughout the history of art, starting from the wall frescoes in Pompeii, throughout the cathedrals, $19^{\text {th }}$-century panoramas up to the IMAX cinemas, and now VR. Oliver Grau states that "the principle of immersion is used to withdraw the apparatus of the medium 
of illusion from the perception of the observers to maximize the intensity of the message being transported. The medium becomes invisible" $(2003,349)$. Similarly, Allison Griffiths defines immersion as "that sense of being in closer communion with something other than the here and now, something that takes us into a virtual reality" (2008). Both of them define the latest cinematic medium IMAX and especially "computer-aided" virtual realities, which not only have the total immersive effect but also fulfil the participants' expectation of interactivity, which in other art forms (as in literature) is not possible. The virtual reality experience offered by the head-mounted display offers this sense of transportation by a multisensorial experience that includes not only visual and auditory impressions, but to some extent also the tactile feeling of suddenly being present in a different space. According to Caroline Jones, "the human sensorium has always been mediated. But over the past few decades that condition has greatly intensified. Amplified, shielded, channeled, prosthetized, simulated, irritated - our sensorium is more mediated than ever before" (Jones 2006, 5). The electronically mediated environment however is not able to offer the sense of total liveness. This inability is related to the machine-created environment's failure to replicate a natural environment with a human interface, being more predictable than a natural environment. As Marie-Laure Ryan notes, the technologies have to have the capability of "predictability of the response [which] demonstrates the intelligence of the system. The user must be able to foresee to some extent the result of his gestures, otherwise they would be pure movements and not intent-driven actions" $(1999,123)$. Of course, this does not mean that this kind of systems should not have a certain level of unpredictability (as Ryan also states [1999, 123]), but this is limited only to some "elements of surprise."

Griffiths argues that "while film and television are capable of immersing viewers in the experience, they do not send shivers down the spine unless one is watching a horror film" $(2008,3)$. This horror-like effect can be created in VR productions too by introducing the sense of liveness and unexpected co-presence of another body. According to Grau, those immersive situations that involve "the creation of expanded image spaces experienced polysensorily and interactively, which enable processual situations, promote the trend toward performance. In this way, the categories of game and game theory gain new significance. Thus, in addition to presenting the long and complex tradition of the concept of immersion, it is essential to portray the most recent dynamic changes that have taken place in images, brought forth by the new options of interaction and evolution” $(2003,9)$. The "shivers" caused by unexpected liveness in VR experiences can be enhanced 
by mechanisms that let the viewer get in touch with the illusory experience of the virtual reality only gradually.

While the above described theories are focusing more on the mediums that can create the immersion, Gordon Calleja emphasizes the notion of presence as well: "virtual environments offer a particular form of mediated experience that was not previously possible. Two terms have been formulated in different disciplines to articulate this experiential phenomenon. Technologists, media psychologists, and human-computer interaction researchers, among others, refer to this experience as presence, while humanists and, later, social scientists adopted the metaphor of immersion" $(2011,33)$. This "presence" means the participants' actual presence (or even telepresence while playing video games) but he points out that truly immersive environments should have the characteristic of liveness, which can mainly be achieved by bodily co-presence.

\section{Conclusions}

As these three examples have shown, the seamed character of VR experience is actually enhancing its immersive effect. Immersion becomes a dynamic function that, through the interactive characteristics of the art form, always presents to the viewer a new layer of experience. As Kwastek argues, "interactive art places the action of the recipient at the heart of its aesthetics. It is the recipient's activity that gives form and presence to the interactive artwork, and the recipient's activity is also the primary source of his aesthetic experience" (2013, XVII). Therefore, the participant's capability to always rephrase the state of immersion is part of the artistic process and the aesthetic experience. VR productions can fulfil the expectations of immersion and of interaction if they become hybrid media and use elements of film and performance. The process of interaction can result in various different kinds of emotions in the audience (as their trajectories can always be different), which can also surprise the orchestrators. But the emotions that such VR productions can trigger are often those that the traditional medium of theatre and film cannot produce and which resemble more the effect of video games. This can be pointed out in Bombina Bombast's production, where the audience members are responsible for each others' experience: if they interrupt the VR experience they can have a feeling of guilt or shame. It is therefore no wonder how VR productions are actually more and more used in psychological experiments. Nevertheless, in order to analyse the immersion level of the medium, concepts regarding performance are the most beneficial. Bodily co-presence can 
result in random or unpredictable reaction from the audience, and the autopoietic feedback loop leads thus to the transformation, not only of the VR production, but also a transformation on the side of the creators/orchestrators and that of the audience/participants, who experience moments of intense excitement.

\section{References}

Artaud, Antonin. 1961. The Theater and its Double. New York: Grove Press.

Benford, Steve and Gabriella Giannachi. 2011. Performing Mixed Reality. Cambridge, Massachusetts: MIT Press.

Böhme, Gernot. 1993. Atmosphere as the Fundamental Concept of a New Aesthetics. Thesis Eleven vol. 36: 113-126.

Bolter, Jay David and Richard Grusin. 2000. Remediation. Understanding New Media. Cambridge, Massachusetts: The MIT Press.

Calleja, Gordon. 2011. In-Game: From Immersion to Incorporation. Cambridge, Massachusetts: MIT Press.

Doležel, Lubomír. 1988. Mimesis and Possible Worlds. Poetics Today vol. 9, no. 3: 475-496.

Eco, Umberto. 1984. The Role of the Reader: Explorations in the Semiotics of Texts. Bloomington: Indiana UP.

Fischer-Lichte, Erika. 2008. The Transformative Power of Performance. A New Aesthetics. London: Routledge.

Foucault, Michel. 1988. Technologies of the Self. Cambridge, Massachusetts: The MIT Press.

Grau, Oliver. 2003. Virtual Art. From Illusion to Immersion. Cambridge, Massachusetts; London, England: The MIT Press.

Graver, David. 2016. Miami Art Week 2016: Theater+Virtual Reality Collide. http://www.coolhunting.com/culture/punchdrunk-vr-experience-samsung. Last accessed 21. 07. 2019.

Griffiths, Alison. 2008. Shivers Down Your Spine - Cinema, Museum, and the Immersive View. New York: Columbia University Press.

Jones, A. Caroline, ed. 2006. Sensorium - Embodied Experience in Technology and Contemporary Art. London: MIT Press.

Kwastek, Katja. 2013. Aesthetics of Interaction in Digital Art. Cambridge, Massachusetts; London, England: MIT Press. 
Milk, Chris. 2015. How Virtual Reality Can Create The Ultimate Empathy Machine. https://www.ted.com/talks/chris_milk_how_virtual_reality_can_ create_the_ultimate_empathy_machine. Last accessed 21.07. 2019

Rouse, Rebecca. 2016. Media of Attraction: A Media Archeology Approach to Panoramas, Kinematography, Mixed Reality and Beyond. In Interactive Storytelling. ICIDS 2016. Lecture Notes in Computer Science, vol. 10045, eds. Francis Nack and Andrew Gordon, 97-107. Cham: Springer.

Ryan, Marie-Laure. 1999. Immersion vs. Interactivity: Virtual Reality and Literary Theory. SubStance vol. 28, no. 2: 110-137.

Salter, Chris. 2017. Technologies of Sense. Exploring the Sensorium in the Age(s) of Immersion. In Limits of Knowing, eds. Thomas Oberender and Joanna Petkiewicz, 174-183. Berlin: Berliner Festspiele/Immersion and Kerber Verlag. 